\title{
ANALYTICAL-NUMERICAL STUDIES OF HYDRODYNAMIC PRESSURES ON SLOPPING DAMS AND VERTICAL DAMS WITH INCLINED RESERVOIR BOT- TOM
}

\author{
D. O. T. Belo ${ }^{1}$, P. M. V. Ribeiro ${ }^{2}$
}

${ }^{1}$ Researcher/Student, Department of Civil Engineering, Federal University of Pernambuco UFPE (corresponding author, danilo_1608@hotmail.com)

${ }^{2}$ Professor, Department of Civil Engineering, Federal University of Pernambuco

\begin{abstract}
Although still present in the popular imagination, the idea that the Brazilian territory is devoid of earthquakes and tradition that structures designed in this region do not require special considerations on this phenomenon are losing strength. Standards for the seismic design of structures were recently developed and reports of seismic phenomena in the country are not rare. The Brazilian seismic activity is low but not non-existent. Earthquakes of significant magnitudes have been recorded and seismic history of this region is still little known. Greater understandings of seismology, the availability of new records of earthquakes and seismic observations of the behavior of structures have shown that a strong earthquake could induce a significant dynamic amplification, capable of possible structural damage in concrete dams. The evaluation of seismic safety of dams is a growing concern, because accidents involving this type of structure have catastrophic consequences, with human and material losses. This paper provides contributions to an analytical-numerical study on the damreservoir interaction problem. The separation of variables method is employed in the closed form solution of the bidimensional Laplace equation over rectangular domains, assuming an infinite reservoir in the longitudinal direction. On a next step, having the analytical expressions as a reference, a finite difference scheme is employed in non-rectangular domains, using the commercial software Excel. These solutions are readily applied in the investigation of hydrodynamic pressures for non-vertical dam-reservoir interface as well as inclined reservoir bottom. For these particular cases, where there is no analytical solution, parametric studies were made, providing simplified expressions and design abacuses. The proposed expressions can be readily applied in practical analysis of dam-reservoir interaction problems.
\end{abstract}

Keywords: Hydrodynamic Pressures, Finite Differences, Laplace Equation, Slopping Dams.

\section{INTRODUCTION}

In the current economic scenario, in which Brazil has a prominent role, it is important not only to promote the works of infrastructure, but also the modernization and upgrading of 
design procedures towards new realities of project. The seismic effect, contrary to what one might imagine, exerts important influence on major constructions such as dams and its consideration has been taking importance with the emergence of specific standards for earthquake-resistant designs [1]. Therefore, the need for studies on the influence exerted by dynamic effects arises as an important analysis case, with a particular field concerned specifically with the study of hydrodynamic pressures. There are classical studies which concern the analytical evaluation of the dam-reservoir interaction, like Westergaard [2] and Chopra [3]. In Brazil, related studies began to appear more recently, like the work of Silva [4], Ribeiro [5-6], Ribeiro et al [7], among others. The results obtained in studies cited above do not, however, consider the inclination of the reservoir or the inclination of the upstream slope and its corresponding effects on the hydrodynamic pressures. Except for a few foreign articles, [8] and [9], nothing has been done in this area domestically. Thus, due to its obvious practical importance and the lack of literature in the area, this study proposes a methodology that analyzes the influence of such factors on the distribution of hydrodynamic pressures in the reservoir for nonrectangular domains using a finite difference scheme as a basis.

\section{ANALYTICAL DEVELOPMENT OF THE GOVERNING EQUATION, BOUN- DARY CONDITIONS AND EXACT SOLUTION.}

\subsection{Analytical development of the governing equation}

The study of phenomena related to the propagation of waves in a continuous elastic medium is based on the wave equation. This equation can be obtained through the equations governing the behavior of the fluids. They are:

$$
\begin{gathered}
\frac{\partial \rho}{\partial \mathrm{t}}+\operatorname{div}(\rho \mathbf{V})=0 ; \text { Continuity equation } \\
\rho \frac{\partial \mathbf{V}}{\partial \mathrm{t}}+\rho \mathbf{V} \operatorname{grad}(\rho \mathbf{V})+\operatorname{grad}(\mathbf{p})+\mu\left[\Delta \mathbf{V}+\frac{1}{3} \operatorname{grad}(\operatorname{div}(\mathbf{V}))\right]=0 ; \text { Navier-Stokes equation } \\
\mathrm{f}(\mathrm{p}, \rho)=\text { constant } \rightarrow \mathrm{p}=\rho \mathrm{c}^{2} ; \text { State equation }
\end{gathered}
$$

in the equations above, $\rho$ is fluid density, $\boldsymbol{V}=V_{x} \boldsymbol{i}+V_{y} \boldsymbol{j}+V_{z} \boldsymbol{k}$ is the speed of flow, $\operatorname{div}(\boldsymbol{V})=$ $\nabla \cdot \boldsymbol{V}=\frac{\partial V_{x}}{\partial x}+\frac{\partial V_{y}}{\partial y}+\frac{\partial V_{z}}{\partial z}, \operatorname{grad}(\boldsymbol{V})=\nabla \boldsymbol{V}=\frac{\partial V}{\partial x} \boldsymbol{i}+\frac{\partial V}{\partial y} \boldsymbol{j}+\frac{\partial V}{\partial z} \boldsymbol{k}, \mathrm{c}$ is the speed of wave propagation in fluid medium and can be calculated for $c=\sqrt{\frac{\kappa}{\rho}}$, where $\kappa$ is the bulk modulus of the fluid volume.

Considering the fluid as compressible and ideal, the Navier-Stokes equation reduces to the Euler equation: 


$$
\rho \frac{\partial V}{\partial t}+\operatorname{grad}(\boldsymbol{p})=0
$$

Differentiating equation (3) and substituting into equation (1), provides:

$$
\operatorname{div}(\boldsymbol{V})=-\frac{1}{\rho c^{2}} \frac{\partial p}{\partial t}
$$

Applying the divergence in equation (4) and replacing the result in equation (5) yields the following equation:

$$
\nabla^{2} p=\frac{1}{c^{2}} \ddot{p}
$$

Equation (6) is exactly the Wave Equation, which estimates the distribution of fluid pressures in a linearly compressible, homogeneous, with small variations in density and small displacements. It should be noted that in equation (6) the operator $\nabla^{2}$ is the three-dimensional Laplacian given by $\nabla^{2}=\frac{\partial^{2}}{\partial x^{2}}+\frac{\partial^{2}}{\partial y^{2}}+\frac{\partial^{2}}{\partial z^{2}}$.

In the case of an incompressible fluid, where the volumetric stiffness becomes infinitely large and therefore, $\mathrm{c} \rightarrow \infty$, equation (6) reduces to the following expression:

$$
\nabla^{2} p=0
$$

Equation (7) is known as Laplace's Equation, related to the pressure field in an incompressible fluid and will be applied throughout this paper.

\subsection{Development of boundary conditions}

Having the equations that govern the distribution of hydrodynamic pressures in a fluid, the next step is given by the development of boundary conditions governing the problem.

\section{- Fluid-Structure Interface:}

Arises due to the compatibility between the movement of the adjacent structure and the contained fluid. Consider Figure 1, which indicates a one-dimensional tube associated with a piston. The movement of the structure $(\bar{u})$ occurs along the axis $\mathrm{x}$ and pressure along a cross-section is constant.

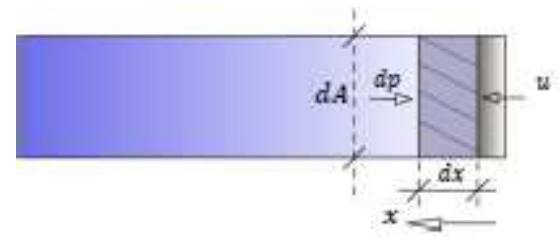

Figure 1. Schematic representation of fluid-structure condition

The dynamic equilibrium of horizontal forces on the fluid element hatched provides: 


$$
\sum F_{x}=m a \quad \therefore \quad-d p d A=d x d A \rho_{f} \bar{u}
$$

where $\rho_{f}$ indicates the density of the fluid. This last equation results in:

$$
\frac{d p}{d x}=-\rho_{f} \bar{u}
$$

which may be extended, resulting in a boundary condition, fluid-structure:

$$
\frac{d p}{d \vec{n}}=-\rho_{f} \overrightarrow{\vec{u}}
$$

where $\vec{n}$ is the normal direction of the surface.

- Rigid wall condition (Neumann condition):

The dynamic equilibrium of horizontal forces on the hatched fluid element provides:

$$
\sum F_{x}=m a \quad \therefore \quad-d p d A=d x d A \rho_{f} \bar{u}
$$

where $\rho_{f}$ indicates the density of the fluid. For a rigid contour the term $\bar{u}$ vanishes, since the contour is rigid. With this last comment Equation (9) is reduced to:

$$
\frac{d p}{d x}=0
$$

which may be substituted in (11), resulting in the boundary condition of the rigid wall:

$$
\frac{d p}{d \vec{n}}=0
$$

Where $\vec{n}$ is the normal direction of the surface.

- Free surface condition (Dirichlet condition):

At the free surface the domain is taken as undisturbed (neglecting free-surface waves effect), therefore we have $p=0$.

\section{- Infinite reservoir condition:}

Radiation condition is considered, in a simplified manner, with zero hydrodynamic pressure at the reservoir limits, which extends to an infinite length.

\subsection{Exact analytical solution for a vertical upstream face with rectangular reservoir}

With the governing equation and boundary conditions of the problem defined, the next step is given by construction of the exact solution. The problem is simplified significantly with the assumptions of a rigid dam (leading to a constant acceleration along the fluid-structure in- 
terface) and an incompressible fluid, and that is generally a first step solution in the analysis of dam-reservoir interaction (being basic hypotheses of the Pseudo-Static Method). Figure 2 provides a depiction of the analyzed rectangular domain including the prescribed boundary conditions.

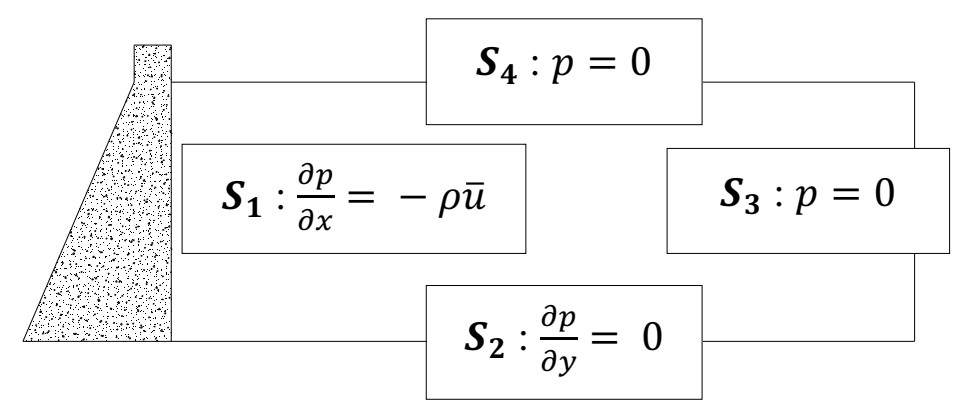

Figure 2. Analyzed rectangular domain and boundary conditions

The governing equation is given by Equation (14):

$$
\nabla^{2} p=0
$$

with $p(x, y)=X(x) Y(y)$ assumed as separable. Therefore, boundary conditions on each direction are uncoupled, resulting in:

$$
\begin{aligned}
& \text { S1: } p_{x}(x, y)=-\rho \bar{u} \text { for } x=0 \\
& \text { S2: } p_{y}(x, y)=0 \text { for } y=0 \\
& S 3: p(x, y)=0 \text { for } x \rightarrow \infty \\
& S 4: p(x, y)=0 \text { for } y=H
\end{aligned}
$$

with $\mathrm{H}$ standing for the reservoir height, which is assumed as equal to the dam's vertical dimension, and $\overline{\mathrm{u}}$ defining the horizontal acceleration of the monolith, assumed as constant along the dam's height.

Using the method of separation of variables to find the expression $\mathrm{p}(\mathrm{x}, \mathrm{y})$ for the given boundary conditions it is assumed a solution as follows:

$$
p(x, y)=X(x) Y(y)
$$

which can be substituted in (14), leading to the following ordinary differential equations:

$$
\begin{aligned}
& X^{\prime \prime}-\lambda X=0 \\
& Y^{\prime \prime}+\lambda Y=0
\end{aligned}
$$

Making up $\lambda=\mu^{2}$, from (21) results: 


$$
\mathrm{Y}(\mathrm{y})=\mathrm{A} \operatorname{sen}(\mu \mathrm{y})+\mathrm{B} \cos (\mu \mathrm{y})
$$

with A and B standing for arbitrary constants. From boundary conditions at S2 and S4 the final solution in y direction is defined by:

$$
\mathrm{Y}_{\mathrm{n}}(\mathrm{y})=\mathrm{B}_{\mathrm{n}} \cos (\mu \mathrm{y})=\mathrm{B}_{\mathrm{n}} \cos \left[(2 \mathrm{n}-1) \frac{\pi}{2} \frac{y}{H}\right]
$$

with $n=1,2,3, \ldots$ given by a positive integer.

The same procedure is reproduced in horizontal direction, $\mathrm{x}$, leading to:

$$
\mathrm{X}(\mathrm{x})=\mathrm{C} \mathrm{e}^{\mu \mathrm{x}}+\mathrm{De} \mathrm{e}^{-\mu \mathrm{x}}
$$

From $\mathrm{p}(\mathrm{x}, \mathrm{y})=0$ for $\mathrm{x} \rightarrow \infty($ condition at $\mathrm{S} 3)$ :

$$
\mathrm{Ce}^{\mu \infty}+\mathrm{De}^{-\mu \infty}=0 \rightarrow \mathrm{C}=0
$$

Therefore, the final solution in $\mathrm{x}$ direction is defined by:

$$
X_{n}(x)=D_{n} e^{-\mu x}=D_{n} e^{-\left[(2 n-1) \frac{\pi}{2 H} x\right]}
$$

Substituting (23) and (26) in equation (19) and considering the Superposition Theorem, which states that the sum of the solutions of a differential equation is also solution of this differential equation:

$$
\mathrm{p}_{\mathrm{n}}(\mathrm{x}, \mathrm{y})=\sum_{\mathrm{n}=1}^{\infty} \mathrm{K}_{\mathrm{n}} \mathrm{e}^{-\mu_{\mathrm{n}} \mathrm{x}} \cos \left(\mu_{\mathrm{n}} \mathrm{y}\right)
$$

with $K_{n}$ standing for a remaining arbitrary constant. Boundary condition at S1 provides the value of this last term and the corresponding final solution is given by:

$$
\mathrm{p}(\mathrm{x}, \mathrm{y})=2 \rho \overline{\mathrm{u}} \mathrm{H} \sum_{\mathrm{n}=1}^{\infty} \frac{(-1)^{\mathrm{n}+1}}{\mu_{\mathrm{n}}^{2}} \mathrm{e}^{-\mu_{\mathrm{n}} \frac{\mathrm{x}}{\mathrm{H}}} \cos \left(\mu_{\mathrm{n}} \frac{\mathrm{y}}{\mathrm{H}}\right)
$$

which provides the hydrodynamic pressure field in an infinite acoustic cavity of height $\mathrm{H}$, subjected to a rigid wall uniform acceleration $\overline{\mathrm{u}}$ at $\mathrm{S} 1$. At this particular position, with $x=0$, the hydrodynamic pressure at the fluid-structure interface is given by:

$$
\mathrm{p}(0, \mathrm{y})=2 \rho \overline{\mathrm{u}} \mathrm{H} \sum_{\mathrm{n}=1}^{\infty} \frac{(-1)^{\mathrm{n}+1}}{\mu_{\mathrm{n}}^{\prime 2}} \cos \left(\mu_{\mathrm{n}} \frac{\mathrm{y}}{\mathrm{H}}\right)
$$

By representing the solution by means of functions of each independent variable, there is a limitation in solving irregular geometries by means of the separation of variables method, since this procedure is limited to rectangular domains. Therefore, obtaining the hydrodynamic pressure field in cases where the boundaries are irregular is only possible by means of numerical methods. It is evident that practical cases may involve slopping upstream faces, slopping reservoir bottom, or even a combination of both. To overcome this problem an outline is proposed using a fictitious boundary at S1. In other words, an extra term is introduced in the formulations $(\cos \theta)$, providing a slopping upstream face, even though this is taken as vertical 
for the application of a standard separation of variables method. Figure 3 explains the proposed scheme.

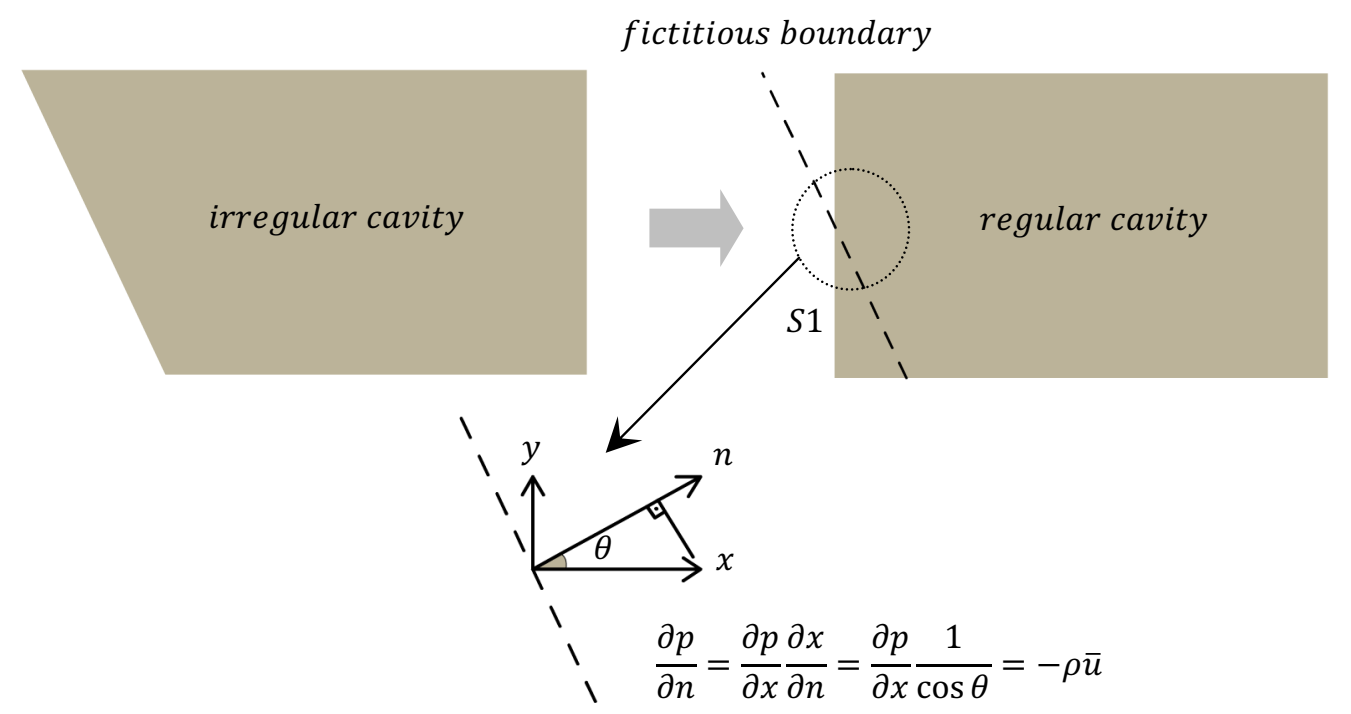

Figure 3. Outline for simplified solution of irregular domains (fictitious boundary)

Therefore, the new boundary condition at S1 using the fictitious boundary is given by:

$$
\frac{\partial p}{\partial x}=-\rho \bar{u} \cos \theta
$$

Using (30) the proposed expression for a slopping upstream face is defined by (31). For $\theta=0$ this latter expression is reduced to (28), resulting in the classical case of a vertical dam.

$$
\mathrm{p}(\mathrm{x}, \mathrm{y})=\left[2 \rho \overline{\mathrm{u}} \mathrm{H} \sum_{\mathrm{n}=1}^{\infty} \frac{(-1)^{\mathrm{n}+1}}{\mu_{\mathrm{n}}^{2}} \mathrm{e}^{-\mu_{\mathrm{n}}} \frac{\mathrm{x}}{\mathrm{H}} \cos \left(\mu_{\mathrm{n}} \frac{\mathrm{y}}{\mathrm{H}}\right)\right] \cdot \cos \theta
$$

\section{NUMERICAL APPROACH TO THE PROBLEM}

Given the limitations of the method of separation of variables and the practical importance of considering both the slopes in the upstream face and reservoir bottom, a numerical analysis of the problem is studied, aiming to verify the influence of such changes in the distribution of hydrodynamic pressures.

For this purpose, a finite difference method is applied, approximating the differential equation at discrete points. A short explanation about the method is discussed below. Consider the diagram in Figure4.

Being " $h$ " the differential step in " $\mathrm{x}$ " axis, approaching the derivative $\mathrm{dy} / \mathrm{dx}$ at point "A" as follows:

$$
\left(\frac{d y}{d x}\right)_{i} \approx \frac{y_{i+1}-y_{i-1}}{2 h}
$$




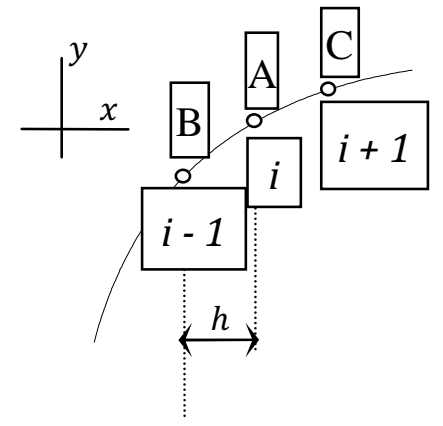

Figure 4. Finite difference scheme

The following is the operator of a second order derivative at the same point:

$$
\left(\frac{d^{2} y}{d x^{2}}\right)_{i} \approx \frac{\frac{y_{i+1}-y_{i}}{h}-\frac{y_{i}-y_{i-1}}{h}}{h} \approx \frac{y_{i+1}-2 y_{i}+y_{i-1}}{h^{2}}
$$

Applying these concepts to the Laplace equation and generalizing them to the "y" axis, we obtain the following expression:

$$
\frac{\partial^{2} p}{\partial x^{2}}+\frac{\partial^{2} p}{\partial y^{2}}=0 \therefore \frac{p_{i+1, j}-2 p_{i, j}+p_{i-1, j}}{h^{2}}+\frac{p_{i, j+1}-2 p_{i, j}+p_{i, j-1}}{k^{2}}=0
$$

Being " $h "$ the step in the axis "x" and "k" the step in "y" axis. Solving the above expression for $p_{i, j}$, we have:

$$
p_{i, j}=\frac{k^{2}\left(p_{i+1, j}+p_{i-1, j}\right)+h^{2}\left(p_{i, j+1}+p_{i, j-1}\right)}{2\left(h^{2}+k^{2}\right)}
$$

\subsection{Mesh example, boundary conditions and linear equations}

Consider the acoustic cavity and the following finite difference mesh, shown on Fig-

\begin{tabular}{|c|c|c|c|c|c|c|c|c|}
\hline 11 & 12 & 13 & 14 & 15 & 16 & 17 & 18 & 19 \\
\hline & 22 & 23 & 24 & 25 & 26 & 27 & 28 & 29 \\
\hline & 32 & 33 & 34 & 35 & 36 & 37 & 38 & 39 \\
\hline 1 & 42 & 43 & 44 & 45 & 46 & 57 & 48 & 49 \\
\hline 51 & 52 & 53 & 54 & 55 & 56 & 57 & 58 & 59 \\
\hline 61 & 62 & 63 & 64 & 65 & 66 & 67 & 68 & 69 \\
\hline 11 & 72 & 73 & 74 & 75 & 76 & 77 & 78 & 79 \\
\hline 81 & 82 & 83 & 84 & 85 & 86 & 87 & 88 & 89 \\
\hline 91 & 92 & 93 & 94 & 95 & 96 & 97 & 98 & 99 \\
\hline
\end{tabular}
ure 5. Because the domain is rectangular, for convenience, we take $\mathrm{h}=\mathrm{k}$. It should be noted that in this way, the value of $p_{i, j}$ is the arithmetic mean values of the four neighbors.

Figure 5. Finite difference mesh (Laplace equation - acoustic cavity) 
To point "34" application of the finite difference operator results in:

$$
p_{34}=\frac{p_{35}+p_{33}+p_{44}+p_{24}}{4}
$$

Application to point "21" provides:

$$
p_{21}=\frac{p_{22}+p_{20}+p_{31}+p_{11}}{4}
$$

It should be noted, however, that the point "20" is not defined in the problem. This point is called "dummy point" and, through the boundary condition of fluid-structure, it can arise in terms of a real component.

Condition $\frac{\partial p}{\partial x}=-\rho \bar{u}$ at $\mathrm{S} 1$ provides:

$$
\frac{p_{22}-p_{20}}{h}=-\rho \bar{u} \therefore p_{20}=p_{22}+2 \rho \bar{u} h
$$

The same procedure can be applied to other points. For those points located at the bottom of the reservoir, proceed analogously.

Application of the differential operator to point"55" gives:

$$
p_{55}=\frac{p_{56}+p_{54}+p_{45}+p_{65}}{4}
$$

Point "65" is not defined and therefore can be obtained by reference to a real component through the rigid wall condition.

From the condition $\frac{\partial p}{\partial y}=0$ at $\mathrm{S} 2$ :

$$
\frac{p_{45}-p_{65}}{h}=0 \therefore p_{65}=p_{45}
$$

In short, operators must be applied to the points in the diagram in Figure 6.

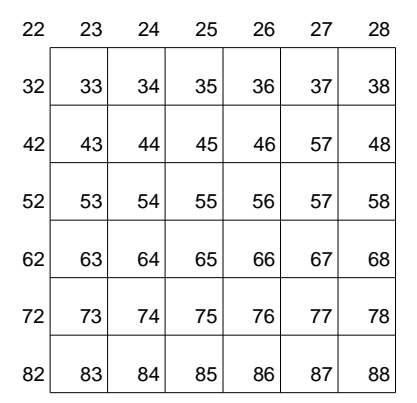

Figure 6. Finite difference mesh including only reference (internal) points 
From what has been said, it can be noticed that the solution through the finite difference method involves obtaining a system of linear equations, the unknowns being the pressures at each point of the reservoir. The differential operator, as shown in Equation (35), is applied only to reference (internal) points. The remaining points at the boundary are related to the interior mesh by means of boundary conditions at S1-S4. The size of such a system is given by the product $\mathrm{m} \times \mathrm{n}$, where " $\mathrm{m}$ " and " $\mathrm{n}$ " are respectively the number of rows and columns of the grid chosen to discretize the domain.

\subsection{Step-by-step solution of a finite difference scheme using Microsoft Excel}

Through this article an iterative routine is proposed, simplifying significantly the problem since, by such approach, there is the no need to solve a system of linear equations. The proposed method consists in setting up an Excel spreadsheet, and the completion of this worksheet consists of the following steps:

1. Fill the boundary conditions at the ends of the field;

2. Enable iterative calculation option;

3. Set Maximum Number of Iterations and Maximum Change;

4. Fill in an internal cell as an average of four neighboring cells (differential operator);

5. "Drag" the cell filled for the remaining internal domain.

Upon completing the above steps, the program will process the iterations according to the increment chosen in step (3) and, after completion of the iterative process, the pressure value at each point is displayed in the respective cell. It is evident that best results are achieved with an increment of rows and columns. However, such operation implies in more computational cost. An example of the foregoing is shown below using a 10 x 10 mesh.

\section{$\underline{\text { Step } 1}$}

- Fluid-structure boundary condition:

By analyzing the points of the fictitious fluid-structure interface, it can be seen that these are functions of real components of the internal domain. With the first being given by the following law, which describes de fluid-structure boundary condition:

$$
p_{i, j}=p_{i, j+2}+2 \rho \bar{u} h
$$

The value of $2 \rho \bar{u} h$ is constant and, for our analysis, the term $\rho \bar{u} h$ was taken as being equal to a unity value. Thus: 


$$
p_{i, j}=p_{i, j+2}+2
$$

The filling of the cells in fluid-structure boundary is illustrated on Figure 7.

\begin{tabular}{|c|c|c|c|c|c|c|c|c|c|c|c|c|}
\hline \multicolumn{3}{|c|}{ SOMA } & \multicolumn{3}{|c|}{$\checkmark \checkmark \times \checkmark f_{x}=\mathrm{C} 2+2$} & \multirow[b]{2}{*}{$\mathrm{F}$} & \multirow[b]{2}{*}{ G } & \multirow[b]{2}{*}{$\mathrm{H}$} & \multirow[b]{2}{*}{ I } & \multirow[b]{2}{*}{ J } & \multirow[b]{2}{*}{ K } & \multirow[b]{2}{*}{ L } \\
\hline 4 & A & B & C & D & $E$ & & & & & & & \\
\hline 1 & & & & & & & & & & & & \\
\hline 2 & $=C 2+2$ & & & & & & & & & & & \\
\hline 3 & & & & & & & & & & & & \\
\hline 4 & & & & & & & & & & & & \\
\hline 5 & & & & & & & & & & & & \\
\hline 6 & & & & & & & & & & & & \\
\hline 7 & & & & & & & & & & & & \\
\hline 8 & & & & & & & & & & & & \\
\hline 9 & & & & & & & & & & & & \\
\hline 10 & & & & & & & & & & & & \\
\hline 11 & & & & & & & & & & & & \\
\hline 12 & & & & & & & & & & & & \\
\hline
\end{tabular}

Figure 7. Fluid-structure boundary condition on Microsoft Excel

- Rigid wall boundary condition:

By analyzing the points on the fictitious fluid-structure interface, it can be seen that these are also functions of the internal domain. These being given by the following law:

$$
p_{i, j}=p_{i-2, j}
$$

The filling of the cell contour for a rigid wall boundary is shown on Figure 8.

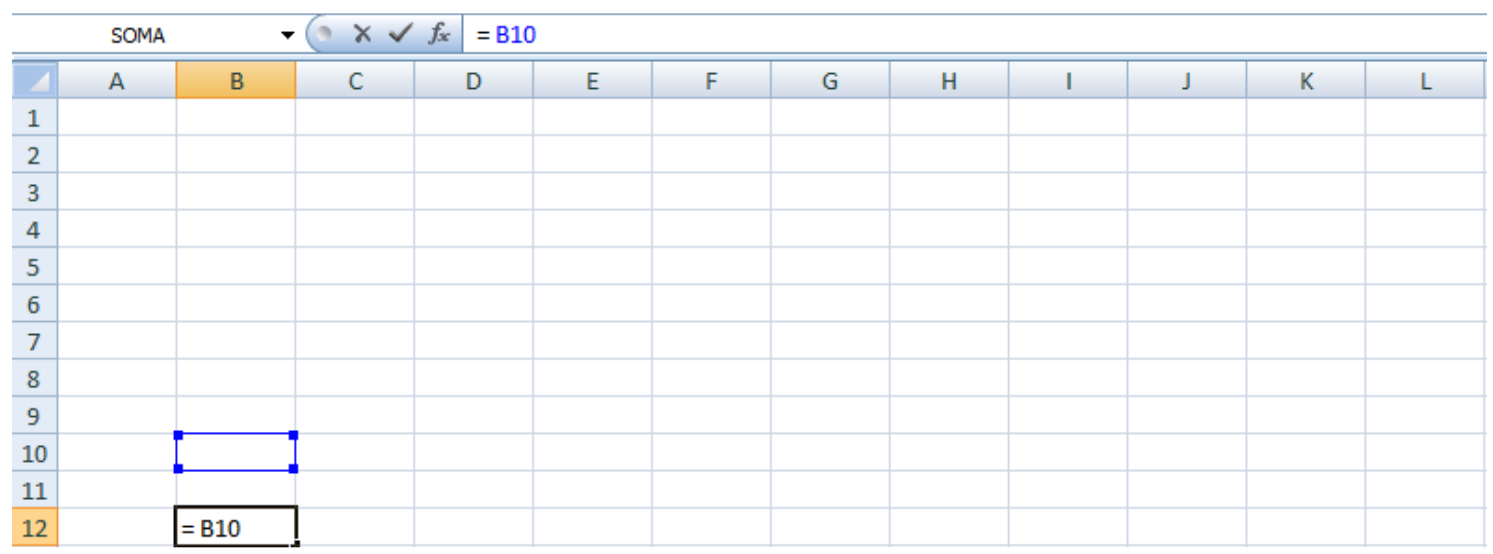

Figure 8. Rigid wall boundary condition on Microsoft Excel

- Free surface and infinite reservoir boundary conditions:

In both cases cells are simply filled with zero. For the discretization chosen including all boundary conditions, the final scheme is shown on Figure 9. 


\begin{tabular}{|c|c|c|c|c|c|c|c|c|c|c|c|c|}
\hline 4 & A & B & C & D & $\mathrm{E}$ & $\mathrm{F}$ & G & $\mathrm{H}$ & I & $\mathrm{J}$ & $\mathrm{K}$ & L \\
\hline 1 & & 0 & 0 & 0 & 0 & 0 & 0 & 0 & 0 & 0 & 0 & \\
\hline 2 & 2 & & & & & & & & & & & 0 \\
\hline 3 & 2 & & & & & & & & & & & 0 \\
\hline 4 & 2 & & & & & & & & & & & 0 \\
\hline 5 & 2 & & & & & & & & & & & 0 \\
\hline 6 & 2 & & & & & & & & & & & 0 \\
\hline 7 & 2 & & & & & & & & & & & 0 \\
\hline 8 & 2 & & & & & & & & & & & 0 \\
\hline 9 & 2 & & & & & & & & & & & 0 \\
\hline 10 & 2 & & & & & & & & & & & 0 \\
\hline 11 & 2 & & & & & & & & & & & 0 \\
\hline 12 & & 0 & 0 & 0 & 0 & 0 & 0 & 0 & 0 & 0 & 0 & \\
\hline
\end{tabular}

Figure 9. Final scheme including all boundary conditions (Step 1)

It should be noted that as the cells of the internal domain were not filled, the conditions of fluid-structure and rigid wall were arbitrated by the program to be zero and two, respectively.

$\underline{\text { Steps } 2 \text { and } 3}$

Enabling iterative calculation can be made as follows:

\section{EXCEL OPTIONS > FORMULAS > ENABLE ITERATIVE CALCULATION > SELECT MAXIMUM NUMBER OF ITERATIONS AND CHANGES}

This is a critical step, because without it Excel displays an error related to circular references. The maximum number of iterations and changes can be chosen according to the desired accuracy by the user and determines also the processing time required.

\section{$\underline{\text { Step } 4}$}

Figure 10 indicates an application example of the differential operator to the internal domain.

\begin{tabular}{|c|c|c|c|c|c|c|c|c|c|c|c|c|}
\hline & SOMA & $\checkmark$ & $x \checkmark$ & $=(\mathrm{C} 2$ & $2+B 1$ & )/4 & & & & & & \\
\hline 4 & A & B & C & D & $E$ & $\mathrm{~F}$ & G & $\mathrm{H}$ & 1 & J & $\mathrm{K}$ & L \\
\hline 1 & & 0] & 0 & 0 & 0 & 0 & 0 & 0 & 0 & 0 & 0 & \\
\hline 2 & 2] & $+\mathrm{B} 3) / 4$ & & & & & & & & & & 0 \\
\hline 3 & 2 & & & & & & & & & & & 0 \\
\hline 4 & 2 & & & & & & & & & & & 0 \\
\hline 5 & 2 & & & & & & & & & & & 0 \\
\hline 6 & 2 & & & & & & & & & & & 0 \\
\hline 7 & 2 & & & & & & & & & & & 0 \\
\hline 8 & 2 & & & & & & & & & & & 0 \\
\hline 9 & 2 & & & & & & & & & & & 0 \\
\hline 10 & 2 & & & & & & & & & & & 0 \\
\hline 11 & 2 & & & & & & & & & & & 0 \\
\hline 12 & & 0 & 0 & 0 & 0 & 0 & 0 & 0 & 0 & 0 & 0 & \\
\hline
\end{tabular}

Figure 10. Differential operator applied to cell B2 (Step 4)

\section{$\underline{\text { Step } 5}$}


Having filled up the first cell as the average of four neighboring cells, we drag it vertically and horizontally so that the same command is repeated in the other cells. When this procedure ends, Excel automatically begins the iterative process which generally lasts a few seconds and ends with the pressure obtaining in each point of the chosen mesh. Figure 11 shows the results obtained with the boundary conditions given on Figure 9 using the $10 \times 10$ mesh. The "dummy points" and the boundary conditions were hatched in order to distinguish them from the internal domain.

\begin{tabular}{|c|c|c|c|c|c|c|c|c|c|c|c|c|}
\hline 4 & A & B & C & D & E & $\mathrm{F}$ & G & H & I & J & K & L \\
\hline 1 & & 0 & 0 & 0 & 0 & 0 & 0 & 0 & 0 & 0 & 0 & \\
\hline 2 & 3,406135 & 2,04274 & 1,406136 & 1,042745 & 0,801222 & 0,623067 & 0,481967 & 0,364268 & 0,261918 & 0,169585 & 0,083347 & 0 \\
\hline 3 & 4,539062 & 3,358695 & 2,539065 & 1,963626 & 1,53908 & 1,209082 & 0,940534 & 0,71319 & 0,513819 & 0,333078 & 0,163802 & 0 \\
\hline 4 & 5,427804 & 4,31392 & 3,427809 & 2,733623 & 2,182396 & 1,733653 & 1,357903 & 1,034144 & 0,747093 & 0,485107 & 0,238783 & 0 \\
\hline 5 & 6,124631 & 5,041383 & 4,124637 & 3,36067 & 2,723238 & 2,18524 & 1,723286 & 1,318397 & 0,955304 & 0,621475 & 0,306226 & 0 \\
\hline 6 & 6,668693 & 5,602357 & 4,668699 & 3,861192 & 3,164656 & 2,560792 & 2,031612 & 1,560858 & 1,134258 & 0,739267 & 0,364646 & 0 \\
\hline 7 & 7,086618 & 6,030668 & 5,086626 & 4,250758 & 3,513413 & 2,86167 & 2,281522 & 1,759174 & 1,281606 & 0,836694 & 0,413091 & 0 \\
\hline 8 & 7,396384 & 6,34709 & 5,396392 & 4,541817 & 3,776584 & 3,090964 & 2,473642 & 1,912717 & 1,396304 & 0,912815 & 0,451024 & 0 \\
\hline 9 & 7,610046 & 6,564932 & 5,610055 & 4,74355 & 3,960154 & 3,251975 & 2,609378 & 2,021757 & 1,478084 & 0,967243 & 0,478193 & 0 \\
\hline 10 & 7,735353 & 6,692558 & 5,735362 & 4,862191 & 4,068524 & 3,347416 & 2,690148 & 2,086858 & 1,527038 & 0,999886 & 0,494505 & 0 \\
\hline 11 & 7,776654 & 6,734604 & 5,776664 & 4,901345 & 4,104352 & 3,379031 & 2,716954 & 2,108498 & 1,543332 & 1,01076 & 0,499942 & 0 \\
\hline 12 & & 6,692558 & 5,735362 & 4,862191 & 4,068524 & 3,347416 & 2,690148 & 2,086858 & 1,527038 & 0,999886 & 0,494505 & \\
\hline
\end{tabular}

Figure 11. Problem solved - end of iteraction procedure (Step 5)

For cases in which the inclination of the reservoir is considered, it proceeds analogously to what was seen. The only changes to such cases are:

1. Will be added cosine and sine terms to boundary conditions of fluid-structure and rigid wall, respectively, since these are defined through the direction normal to the surface;

2. The values of $p(i, j)$ are obtained from equation (35), and increments " $h "$ and " $k$ " will vary according to the inclination of the reservoir. So, for example, an inclination of $\alpha=30^{\circ}$ of the upstream slope implies in $\theta=60^{\circ}$, and thus having $\mathrm{k}=1$, we have $\mathrm{h}=1.73205$. Figure 12 clarifies this interpretation.

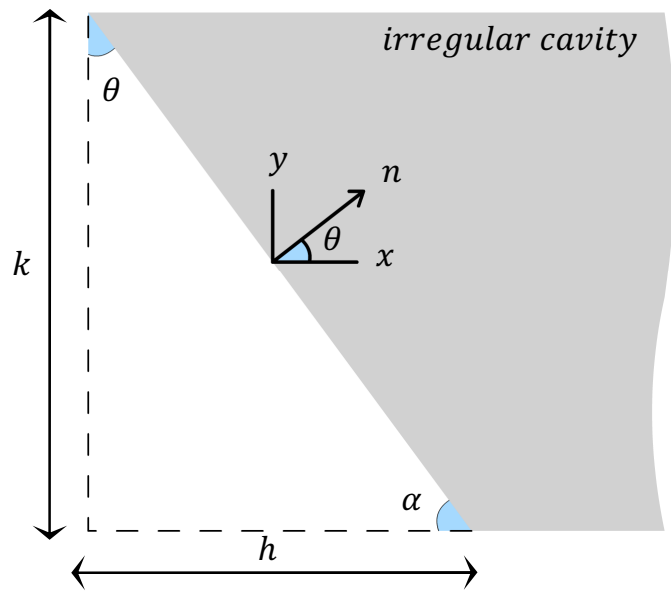

Figure 12. Upstream inclined reservoir with defined angles and dimensions 


\section{CONVERGENCE STUDY - ANALYTICAL X NUMERICAL}

To validate the proposed methodology a comparison was made between the distribution of pressures through the analytical formulation and the numerical results. The latter being analyzed by three different mesh schemes, in order to verify the influence of its size in the refinement of the results. Figure 13 presents these analyses.

From the graphic analysis it is concluded that the mesh size varies the numerical results. Mesh 20 x 30 gives good results with a decreasing ratio y / $\mathrm{H}$, and those are the values that matter most to analysis, with an overall minor error when compared to the rectangular (analytical) solution. Therefore, this mesh was chosen for the proceedings analyses.

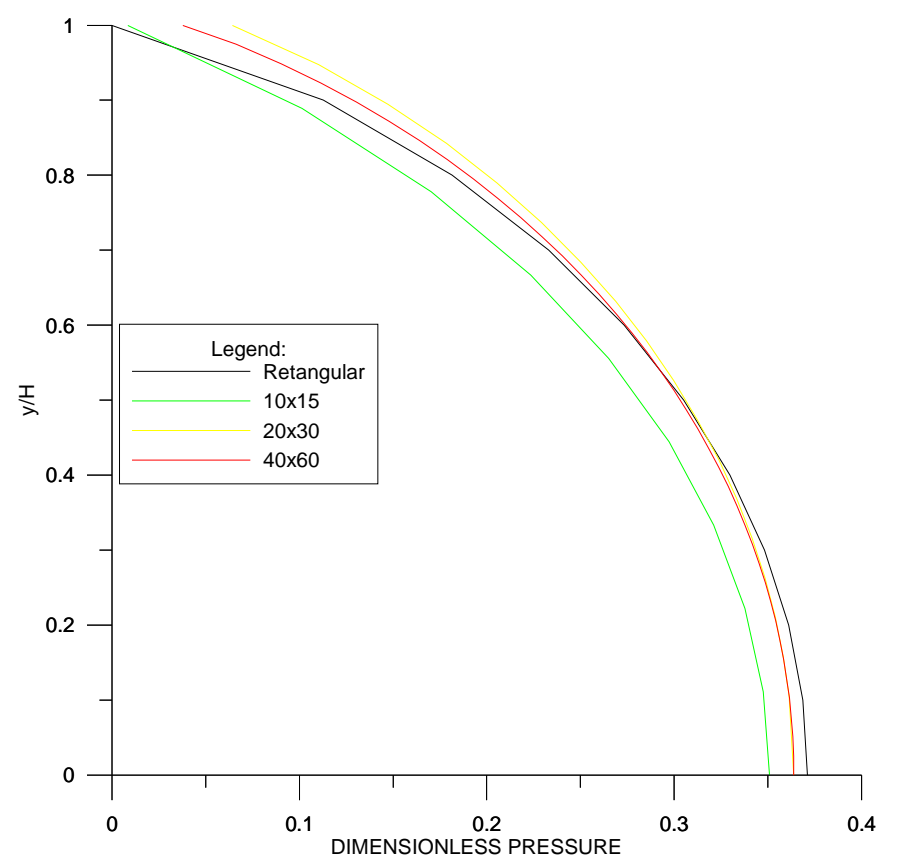

Figure 13. Dimensionless pressure at fluid-structure interface - analytical x numerical

\section{APPLICATION EXAMPLES AND RESULTS}

5.1. Influence of the upstream slope on the hydrodynamic pressure distribution

To evaluate the importance of the upstream slope on the pressure distribution along the fluid-structure interface, analyses were performed for three different numerical models, including angles of 30, 45 and 60 degrees. Results are presented on Figure 14.

It is clear from the graphical analysis results that an increment of the upstream slope results in overall greater pressure values at the fluid-structure interface. The "saws" on the charts representing the hydrodynamic pressures tend to disappear with a more refined discretization of the reservoir. It should also be noted that maximum values are not at base of the dam (in contrast to a vertical dam). 


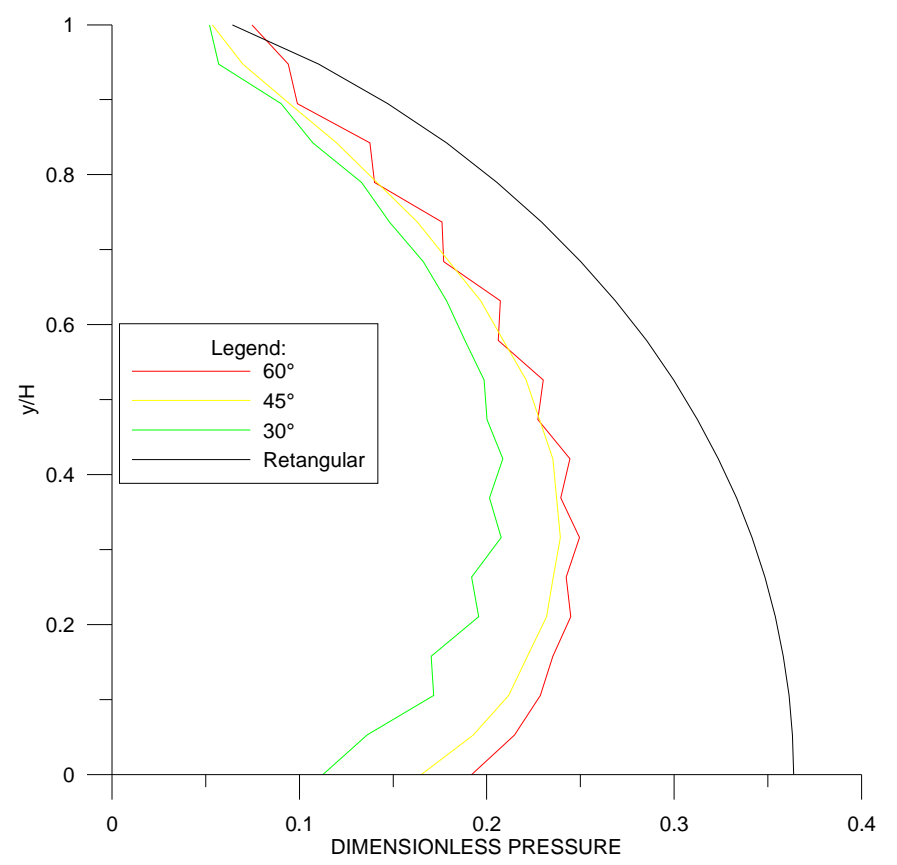

Figure 14. Dimensionless pressure at fluid-structure interface - slopping face

Another analysis of practical importance is given by an error estimative of proposed expression (31) when compared to results provided by a numerical analysis. Figures 15, 16 and 17 present these results.

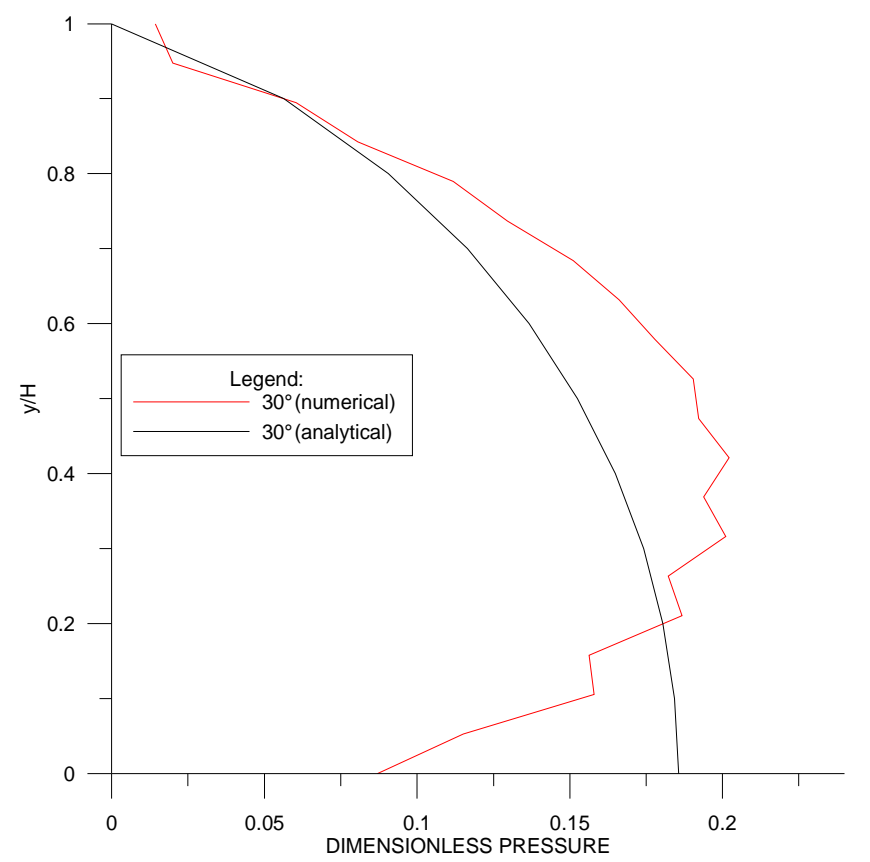

Figure 15. Results for a 30degrees upstream slope (analytical x numerical)

Through the graphical analysis, one can see that for values of y / $\mathrm{H}$ between 0.4 and 0.6 the values obtained by the proposed analytical expression and the corresponding finite difference scheme approach as the angle of the upstream slope decreases. Moreover, for values of $y / H$ less than 0.2 the values of the pressures in both methods differ, with the analytical values oversized. Still, the proposed analytical formulation emerges as an additional tool for the preliminary assessment of pressures on slopping dams. 


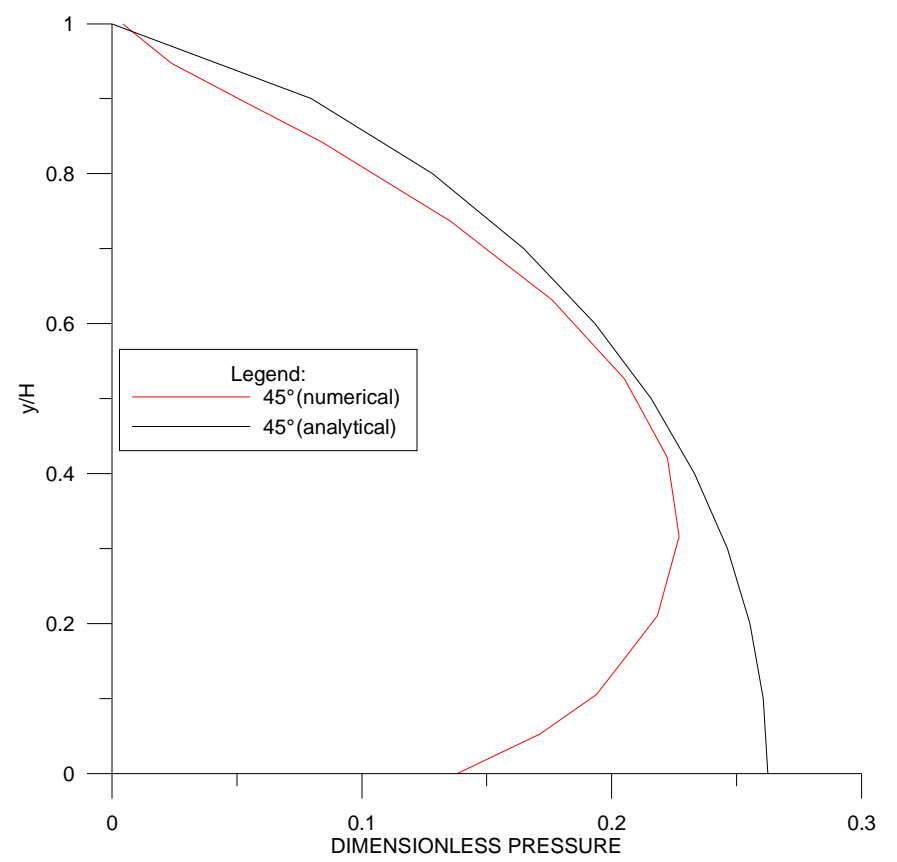

Figure 16. Results for a 45 degrees upstream slope (analytical x numerical)

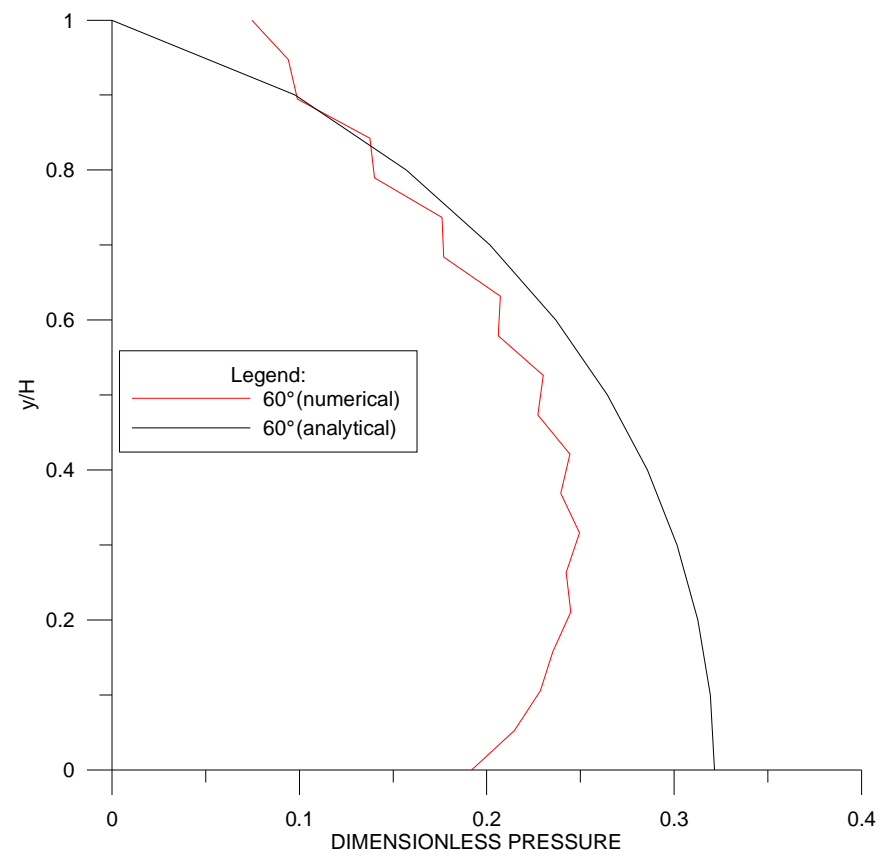

Figure 17. Results for a 60 degrees upstream slope (analytical x numerical)

5.2. Influence of the reservoir bottom slope on the hydrodynamic pressure distribution

Evaluation of the of the reservoir bottom slope effects on the distribution of fluid pressure at the interface was performed for three different numerical models, with variation made for angles of 30-60 degrees, with an increment of 15 degrees. Figure 18 presents these results.

It is clear from the graphical analysis that as the inclination of the tank bottom increases, the greater the pressure for values of $\mathrm{y} / \mathrm{H}$ between 0.4 and 0.6 . The pressure for values of 
$\mathrm{y} / \mathrm{H}$ less than 0.1 follows the inverse order. That is, the pressure at the base of the dam decreases with an increasing inclination of the reservoir bottom.

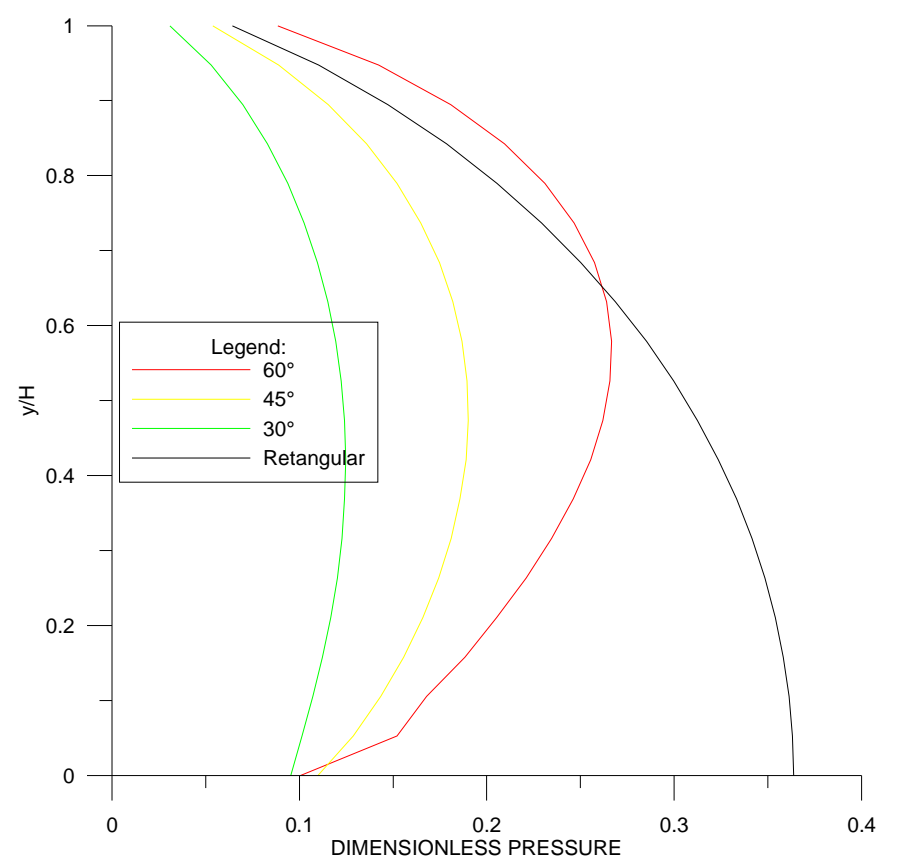

Figure 18. Dimensionless pressure at fluid-structure interface - slopping bottom

\section{CONCLUDING REMARKS}

A simple and practical method was made to evaluate the distribution of pressures along the fluid-structure interface considering different geometries of the domain, namely, the slope of the ornament on the upstream side and the inclination of the reservoir bottom.

It could be seen that in both cases there was a significant shift in the distribution of pressures and that the value of hydrodynamic pressures at the bottom of the reservoir decreases as the angle of inclination decreases for both the upstream face and the reservoir bottom slope.

The major advantage of applying the finite difference method by using the commercial program Excel is to obtain the pressure values directly, without the need to solve a linear system of equations. This same procedure can be applied to other problems for solution of differential equations separate and / or including other boundary conditions. Therefore, the proposed procedure is an ideal introductory tool for young researchers working with finite differences schemes.

The analytical formulation proposed for cases with an upstream slope, despite its physical and mathematical sense, emerges as an alternative for practical problems dealing with limitations imposed by traditional theory, which applied are only to vertical dams. Despite the errors, the proposed formulation provides a good estimative of pressure values at the dam-reservoir interface. 


\section{ACKNOWLEDGMENTS}

The authors thank CNPq for the financial resources (grants) received for this work.

\section{REFERENCES}

[1] NBR15421. Design of Earthquake Resistant Structures - Procedure. Brazilian Association of Technical Standards - ABNT, 2006.

[2] Westergaard, HM (1933) - "Water Pressures on dams During earthquakes." ASC Transactions, 59 (8), Part 3, p. 418-472.

[3] CHOPRA, A. K. (1978) - "Earthquake resistant design of concrete gravity dams." Journal of the Structural Division, ASCE, Vol 104, pp. 953-971.

[4] Silva, S. F. "Dam-Reservoir Interaction Dynamics: Analytical and Numerical Models", Doctoral Thesis in Structures and Construction, Publication E.TD-05A/07, Department of Civil and Environmental Engineering, University of Brasília, Brasília, DF, 220 p., 2007.

[5] RIBEIRO, P. M. V. An Analytical Methodology for Evaluation of Stress Field in Concrete Gravity Dams during Earthquakes. 162p.Dissertation (Master in Structures and Building) Department of Civil and Environmental Engineering, University of Brasilia, Brasilia, 2006.

[6] Ribeiro, P. M. V., 2010, "Analytical Solutions for Two-Dimensional Acoustic Cavities with Application to the Study of Dam-Reservoir Interaction Dynamics", PhD Thesis, Publication E.TD-004A/10, Department of Civil and Environmental Engineering, University of Brasilia, Brasilia, DF, 306 p.

[7] RIBEIRO, P. M. V., Melo, C. A. E., Pedroso, L. J. "Semi-Analytical Solution of DamReservoir Interaction in the Fundamental Mode Shape." In: Solid Mechanics in Brazil 2009. New York: ABCM - Brazilian Association of Mechanical Sciences and Engineering, 2009, p. 445-473.

[8] AVILÉS, Javier, LI, Xiangyue. "Analytical-Numerical Solution for Hydrodynamic Pressures on Dams with Sloping Face Considering Compressibility and Viscosity of Water." Mexican Institute of Technology del Agua.

[9] Chwang, Allen T. "Hydrodynamic Pressures on Dams During Earthquakes Sloping. Part 2. Exact Theory. " Division of Applied Science and Engineering, California Institute of Technology.

[10] Microsof Excel 2007. User Manual.

[11] Grapher v. 7.0. User Manual. 